\title{
Comparison of the conformational dynamics of structurally different AP-endonucleases APE1 and Nfo during AP-endonuclease activity
}

\author{
Senchurova S. ${ }^{1,2 *}$, Kuznetsova A. ${ }^{1}$, Ishchenko A. ${ }^{3}$, Saparbaev M. ${ }^{3}$, \\ Fedorova O. ${ }^{1}$, Kuznetsov N. ${ }^{1}$ \\ ${ }^{1}$ Institute of Chemical Biology and Fundamental Medicine, SB RAS, Novosibirsk, Russia \\ ${ }^{2}$ Novosibirsk State University, Department of Natural Sciences, Novisibirsk, Russia \\ ${ }^{3}$ Université Paris-Saclay, Group Mechanisms of DNA Repair and Carcinogenesis, Equipe Labellisée \\ LIGUE 2016, CNRS UMR9019, Villejuif, France \\ *svetasenchurova@gmail.com
}

Key words: DNA repair, apurinic/apyrimidinic endonuclease, damaged DNA, abasic site, conformational dynamics, stopped-flow enzyme kinetics

Motivation and Aim: Apurinic/apyrimidinic (AP) endonucleases Nfo (Escherichia coli) and APE1 (human) represent two conserved structural families of enzymes that cleave AP-site-containing DNA in base excision repair. Nfo and APE1 have completely different structures of the DNA-binding site, catalytically active amino acid residues and catalytic metal ions. Nonetheless, both enzymes induce DNA bending, AP-site backbone eversion into the active-site pocket and extrusion of the nucleotide located opposite the damage. All these stages may depend on local stability of the DNA duplex near the lesion.

Methods and Algorithms: We analysed effects of natural nucleotides located opposite a lesion on catalytic complex formation stages and DNA cleavage efficacy. Several model DNA substrates that contain an AP-site analogue [F-site, i.e., (2R,3S)-2(hydroxymethyl)-3-hydroxytetrahydrofuran] opposite G, A, T or C were used to monitor real-time conformational changes of the tested enzymes during interaction with DNA using changes in the enzymes' intrinsic fluorescence intensity mainly caused by Trp fluorescence. The extrusion of the nucleotide located opposite F-site was recorded via fluorescence intensity changes of two base analogues.

Results: Our stopped-flow Trp data suggest that catalytic-complex formation in most cases proceeds through a two-step kinetic mechanism of binding. These findings permit the conclusion that kinetic parameters of the binding steps resulting in a catalytically competent enzyme-substrate complex are significantly different among the tested F/Nsubstrates, indicating the importance of the opposite-base nature. Initial-DNA-binding constants and total binding constants typically decrease in the order F/G > F/A _ F/T > F/C. The catalytic rate constant slightly depended on the opposite-nucleotide nature.

Conclusion: Thus, structurally different AP endonucleases Nfo and APE1 utilise a common strategy of damage recognition controlled by enzyme conformational transitions after initial DNA binding.

Acknowledgements: This work was funded by Russian Science Foundation grant No. 19-74-10034. 